\title{
Non-Weighted Evaluation Function in Multi-Objective Problems
}

\author{
Bayadir Abbas Himyari ${ }^{1, *}$, Azman Yasin ${ }^{2}$, Horizon Gitano ${ }^{3}$ \\ ${ }^{1}$ Universiti Utara Malaysia (UUM) \\ Kedah, Malaysia \\ ${ }^{2}$ University of Babylon \\ Babylon, Iraq \\ Horizon Gitano ${ }^{c}$, \\ ${ }^{3}$ University Kuala Lumpur Malaysian Spanish Institute (UNIKL MSI) \\ Kedah, Malaysia, \\ ${ }^{*}$ Corresponding author's email: bayadir_abbas [AT] yahoo.com
}

\begin{abstract}
An evaluation function is proposed to deal with multi-objective problems without weight using a new composition method. Improving the evaluation function by reducing its complexity through discarding the weights. The evaluation function is utilized for the optimization of fuzzy rules.

A genetic algorithm is applied as a multi-objective algorithm for fuzzy rules extraction. Simplicity during building fuzzy inference system and reducing the computational complexity is required. The algorithm is applied on AFR data sets.
\end{abstract}

Keywords--- Multi-objective Problems, Non Weighted Evaluation Function, Fuzzy Systems, Genetic Algorithms, Rule extraction, Air/Fuel Ratio Data

\section{INTRODUCTION}

A rational resolution to a multi-objective issue is to study a set of solutions, with each of it fulfilling the goals at a satisfactory level without being dominated by any other resolution. GAs are a common meta-heuristic especially compatible with these kind of problems. Traditional GAs are tailored to accommodate multi-objective issues by utilising specialised fitness functions and launching methods to endorse solution diversity.

The key drive behind using genetic algorithms in the detection of high-level prediction rules is to carry out a global search and deal with attribute interaction in a better manner.

The creation of a fuzzy rule base (RB) comprises the number of antecedents in the condition part of every rule, the number of fuzzy rules, and the rule class in the consequent part of each rule, which in turn can lead to an increase in complexity and inaccuracy of the end system. Conventionally, models with high syntactic complexity tend to be highly accurate, while models with low syntactic complexity tend to be low on accuracy. Striking a trade-off between the complexity of the fuzzy RB and the accurateness of the end system will lead to a multi-objective assessment problem, where most of the work in this field will be managed with an aggregate method known as weighted sum (Dehuri, Patnaik, Ghosh, \& Mall, 2008; Gacto, Alcalá, \& Herrera, 2010; Ishibuchi, Nakashima, \& Murata, 2001; Ishibuchi \& Nojima, 2007; Ishibuchi \& Yamamoto, 2004). But there is one inevitable problem of this approach and that is the generation of a set of weights which correctly measures the objectives when nothing is known about the problem, which might be difficult if the number of objectives is high (Mandal \& Pal, 2011; Xu \& Zhou, 2011). These weights are generally identified using a trial and error method.

Tradeoff of accuracy and interpretability is very important. Adding the interestingness is benefit and so important in real world applications to discover hidden rules and information. In this research, a new Composition method will be used to solve this problem.

\section{FUZZY RULE EXTRACTION}

The rule extraction (RE) procedure could be deemed as a transformation of continuous, symbolic, or distinct knowledge from a dataset into beneficial, representative propositional logical rules which elaborate the knowledge embedded in datasets with adequate precision. 
Model structure as FRBSs comprises an extension to conventional rule-based systems, as they deal with "IF-THEN", rules. However, its antecedents and consequents comprise fuzzy logic statements rather than classical ones. Furthermore, with regards to fuzzy sets with linguistic labels, the output system demonstrates a higher level of interpretability for the expert to comprehend the former's working procedure, and the inner particulars of the problem characteristics (Fernández, López, del Jesus, \& Herrera, 2015; Gacto, Alcalá, \& Herrera, 2011).

A fuzzy RB includes several rules stated as:

\section{$\mathrm{R}:$ IF $\boldsymbol{x}$ is $A_{i}$, THEN $\boldsymbol{y}$ is $B i$}

where $A i=\left\{A_{i 1}, A_{i 2}, \ldots, A_{i n}\right\}, B_{i}=B_{i 1}, B_{i 2}, \ldots, B_{i m}$, and $A_{i j}$ and $B_{i k}$ are, respectively, fuzzy sets which outline an input and output space partitioning, $x$ is the input, and $y$ is the output. The condition, also known as premise, comprises several antecedents which are combined by various operators like AND or OR computed with $t$-norms.

\subsection{Assessment Criteria for Rule Extraction}

There are three measurements that are typically employed to assess rule extraction (Huysmans, Baesens, \& Vanthienen, 2006; Huysmans, Dejaeger, Mues, Vanthienen, \& Baesens, 2011). These measurements are as follows:

1. Predictive accuracy. The induction of a rule possessing a considerably high degree of predictive accuracy is a primary consideration in rule inductive learning.

2. Comprehensibility. Another important aspect that should be taken into consideration is the comprehensibility of learned rule sets. To determine comprehensibility, it should be identified whether the extracted (or refined) rule sets could be inspected and elucidated by domain experts.

3. Consistency. The assessment of consistency is likewise related to the rules generated from a learning model. An extracted rule set is found consistent if rule sets that generate the same classification of unseen examples are derived from a learning model, even in different training sessions.

Rule extraction also has the objective of deriving a subset of interesting rules from all those that were identified. Numerous data mining algorithms were designed to identify accurate, comprehensible, and consistent rules, but most of them fail to identify interesting rules, which is a more difficult and challenging task. Approaches to the identification of interesting rules can generally be classified into subjective and objective methods. Objective methods are driven by data and independent of the domain. A number of these approaches compare a discovered rule with other rules instead of user beliefs. Thus, the underlying principle is that a rule's level of being interesting relies on both its quality and like comparability to other rules.

This work employed objective approaches to identify interesting rules as the measure for assessing discovered rules.

\section{METHODOLOGY}

A genetic algorithm of real value has been applied, namely the Iterative Approach, in which each rule is represented by a chromosome. A single rule is derived by the use of the genetic algorithm with iterative.

Where the fitness function is concerned, a 3-objective Genetic algorithm is applied in order to achieve top efficiency of the rule database. It does so by employing a composition method for designing the fitness function using the three appointed targets. The objectives themselves include interpretability level, interestingness level, and quality of the rule (Dehuri et al., 2008; Noda, Freitas, \& Lopes, 1999; Noda, Freitas, \& Yamakami, 2003).

The GA discovers the rules, which were subject to evaluation according to three criteria, namely:

(a) Rule's Quality.

This represents the calculation of the rule quality

$$
Q=\frac{T P}{T P+F N}+\frac{T N}{T N+F P}
$$

Where,

TP stands for the number of examples that are covered by the rule and has the same class from the class that is predicted by the rule,

FP stands for the number of examples that is covered by the rule and has a different class from the class that is predicted by the rule,

FN is the number of examples that is not covered by the rule, but has the same class with the class that is predicted by the rule, and

TN is the number of examples that is not covered by the rule, and has a different class with the class that is predicted by the rule.

(b) Degree of Interpretability (Rule's Complexity)

A value $(\mathrm{K})$ stands for a number of rules and the number of these rules' conditions can be considered for its comprehensibility characteristic. $(\mathrm{K})$ is an inversely proportional value that is relative to the 
number of conditions $\mathrm{N}(\mathrm{X})$ in $\mathrm{X}$, the rule's antecedent. Whether a rule can have a minimum of $\mathrm{M}$ conditions, the comprehensibility can thus be defined as:

$$
K=1-(N(X) / M)
$$

(c) Degree of interestingness (Surprisingness)

In data mining, a key factor is concerned with the discovered knowledge and that it should somehow be interesting; here, the term "interestingness" is arguably related with "surprisingness" (unexpectedness), novelty, and usefulness. To date, there are a number of objective criteria proposed in the measurement of the interestingness of rule. Frietas (1999) proposed one of these criteria based on information theory.

$$
\text { Interestingness }=\left(1 /\left(\sum_{i=1}^{k} \operatorname{InfoGAin}(A i)\right) / k\right)
$$

Where, InfoGain $(A i)$ is the information gain (MI) of the $i$ th attribute occurring in the rule antecedent and $k$ the number of attributes occurring in the rule antecedent.

The purpose of the three objectives here is towards maximising the rule's quality, maximising the degree of interpretability, and maximising the degree of interestingness, as illustrated in Figure 1. A Composition Function method is used to aggregate the three objectives without requiring weights in order to generate a fitness function which best stands for the desirability of the chromosome (or FIS).

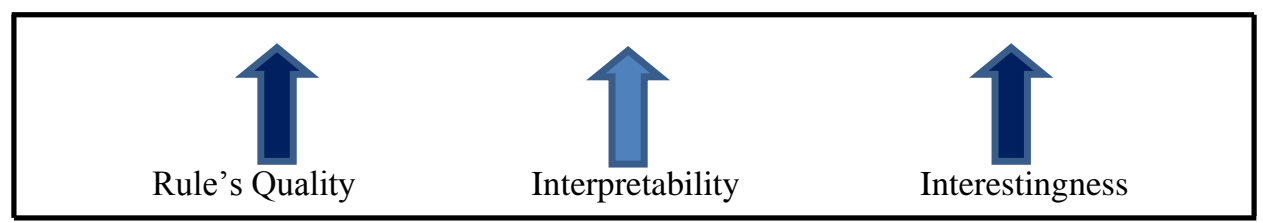

Figure 1. Composition Function of three objectives

A number of the composition functions are used in this work, as,

- $\quad \min (\max$ (Quality, Interpretability), Interestingness)

- $\quad$ probor (min (Quality, Interpretability), Interestingness)

- $\quad$ probor (max (Quality, Interpretability), Interestingness)

Where min stands for the operator (AND) between two values as it takes always the minimum between them, max stands for the operator (OR) between two values as it takes always the maximum, Avg stands for the average, and Probor stands for probabilistic OR.

Figures 2(a), 2(b), and 2(c) illustrates the probor, max, and min function respectively. Where, $y 1, y 2$ are the variables and yy stand for the function concerned.

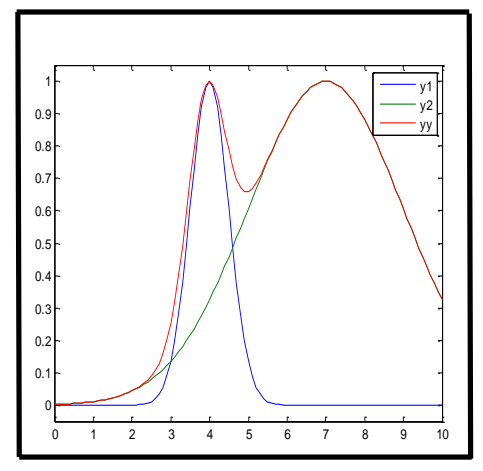

(a)

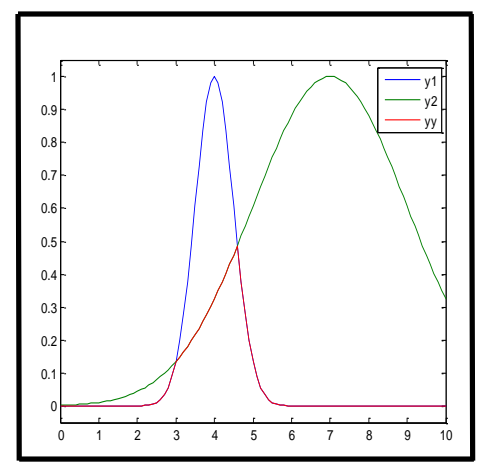

(b)

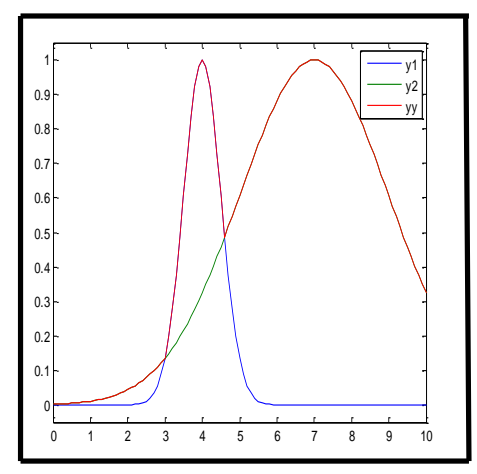

(c)

Figure 2. Probor Function 


\section{MULTI-OBJECTIVE EVALUATION FUNCTION}

Using 3-objective fitness function will improve the optimization process. The objectives are the rule's quality, degree of interpretability, and degree of interestingness. As a multi-objective problem, conjucting these objectives together has the obstacle of using the weights in a weighted sum approach. This research proposed a Composition method to overcome this problem:

- $\quad \min (\max$ (Quality, Interpretability), Interestingness)

- $\quad$ probor (min (Quality, Interpretability), Interestingness)

- $\quad$ probor (max (Quality, Interpretability), Interestingness)

In Table 1, 2,.3 are some examples to show these functions work:

Assuming A= Rule Quality, B= Rule interpretability, $C=$ Rule interestness

Table 1: $\min (\max (A, B), C)$ Examples

\begin{tabular}{lccccc}
\hline $\mathbf{A}$ & $\mathbf{B}$ & $\mathbf{C}$ & $\boldsymbol{m i n}(\mathbf{A}, \mathbf{B})$ & $\boldsymbol{m a x}(\mathbf{A}, \mathbf{B})$ & $\boldsymbol{m i n}(\boldsymbol{m a x}(\mathbf{A}, \mathbf{B}), \mathbf{C})$ \\
\hline 0.6 & 0.5 & 0.3 & 0.5 & 0.6 & $\mathbf{0 . 3}$ \\
0.6 & 0.5 & 0.9 & 0.5 & 0.6 & $\mathbf{0 . 6}$ \\
0.6 & 0.3 & 0.3 & 0.3 & 0.6 & $\mathbf{0 . 3}$ \\
0.6 & 0.3 & 0.5 & 0.3 & 0.6 & $\mathbf{0 . 5}$ \\
0.6 & 0.6 & 0.4 & 0.6 & 0.6 & $\mathbf{0 . 4}$ \\
0.6 & 0.2 & 0.6 & 0.2 & 0.6 & $\mathbf{0 . 6}$ \\
0.3 & 0.9 & 0.1 & 0.3 & 0.9 & $\mathbf{0 . 1}$ \\
0.3 & 0.9 & 0.9 & 0.3 & 0.9 & $\mathbf{0 . 9}$ \\
0.3 & 0.9 & 0.5 & 0.3 & 0.9 & $\mathbf{0 . 5}$ \\
0.3 & 0.1 & 0.9 & 0.1 & 0.3 & $\mathbf{0 . 3}$ \\
0.9 & 0.7 & 0.3 & 0.7 & 0.9 & $\mathbf{0 . 3}$ \\
0.9 & 0.5 & 0.1 & 0.5 & 0.9 & $\mathbf{0 . 1}$ \\
0.4 & 0.8 & 0.5 & 0.4 & 0.8 & $\mathbf{0 . 5}$ \\
0.2 & 0.3 & 0.5 & 0.2 & 0.3 & $\mathbf{0 . 3}$ \\
\hline
\end{tabular}

Table 2: $\operatorname{probor}(\min (A, B), C)$ Examples

\begin{tabular}{lccrrr}
\hline $\mathrm{A}$ & $\mathrm{B}$ & $\mathrm{C}$ & \multicolumn{1}{c}{$\min (\mathrm{A}, \mathrm{B})$} & $\max (\mathrm{A}, \mathrm{B})$ & $\operatorname{probor}(\min (\mathrm{A}, \mathrm{B}), \mathrm{C}$ \\
\hline 0.6 & 0.5 & 0.3 & 0.5 & 0.6 & $\mathbf{0 . 6 5}$ \\
0.6 & 0.5 & 0.9 & 0.5 & 0.6 & $\mathbf{0 . 9 5}$ \\
0.6 & 0.3 & 0.3 & 0.3 & 0.6 & $\mathbf{0 . 5 1}$ \\
0.6 & 0.3 & 0.5 & 0.3 & 0.6 & $\mathbf{0 . 6 5}$ \\
0.6 & 0.6 & 0.4 & 0.6 & 0.6 & $\mathbf{0 . 7 6}$ \\
0.6 & 0.2 & 0.6 & 0.2 & 0.6 & $\mathbf{0 . 6 8}$ \\
0.3 & 0.9 & 0.1 & 0.3 & 0.9 & $\mathbf{0 . 3 7}$ \\
0.3 & 0.9 & 0.9 & 0.3 & 0.9 & $\mathbf{0 . 9 3}$ \\
0.3 & 0.9 & 0.5 & 0.3 & 0.9 & $\mathbf{0 . 6 5}$ \\
0.3 & 0.1 & 0.9 & 0.1 & 0.3 & $\mathbf{0 . 9 1}$ \\
0.9 & 0.7 & 0.3 & 0.7 & 0.9 & $\mathbf{0 . 7 9}$ \\
0.9 & 0.5 & 0.1 & 0.5 & 0.9 & $\mathbf{0 . 1}$ \\
0.4 & 0.8 & 0.5 & 0.4 & 0.8 & $\mathbf{0 . 7}$ \\
0.2 & 0.3 & 0.5 & 0.2 & 0.3 & $\mathbf{0 . 6}$ \\
\hline
\end{tabular}


Table 3: probor(max $(A, B), C)$ Examples

\begin{tabular}{llcrrr}
\hline A & B & C & \multicolumn{1}{c}{$\min (\mathrm{A}, \mathrm{B})$} & $\max (\mathrm{A}, \mathrm{B})$ & $\operatorname{probor}(\max (\mathrm{A}, \mathrm{B}), \mathrm{C})$ \\
\hline 0.6 & 0.5 & 0.3 & 0.5 & 0.6 & $\mathbf{0 . 7 2}$ \\
0.6 & 0.5 & 0.9 & 0.5 & 0.6 & $\mathbf{0 . 9 6}$ \\
0.6 & 0.3 & 0.3 & 0.3 & 0.6 & $\mathbf{0 . 7 2}$ \\
0.6 & 0.3 & 0.5 & 0.3 & 0.6 & $\mathbf{0 . 8}$ \\
0.6 & 0.6 & 0.4 & 0.6 & 0.6 & $\mathbf{0 . 7 6}$ \\
0.6 & 0.2 & 0.6 & 0.2 & 0.6 & $\mathbf{0 . 8 4}$ \\
0.3 & 0.9 & 0.1 & 0.3 & 0.9 & $\mathbf{0 . 9 1}$ \\
0.3 & 0.9 & 0.9 & 0.3 & 0.9 & $\mathbf{0 . 9 9}$ \\
0.3 & 0.9 & 0.5 & 0.3 & 0.9 & $\mathbf{0 . 9 5}$ \\
0.3 & 0.1 & 0.9 & 0.1 & 0.3 & $\mathbf{0 . 9 3}$ \\
0.9 & 0.7 & 0.3 & 0.7 & 0.9 & $\mathbf{0 . 9 3}$ \\
0.9 & 0.5 & 0.1 & 0.5 & 0.9 & $\mathbf{0 . 9 1}$ \\
0.4 & 0.8 & 0.5 & 0.4 & 0.8 & $\mathbf{0 . 9}$ \\
0.2 & 0.3 & 0.5 & 0.2 & 0.3 & $\mathbf{0 . 6 5}$ \\
\hline
\end{tabular}

Considering the above tables, can notice the following:

- $\min (\max (\mathbf{A}, \mathbf{B}), \mathbf{C})$ : does not take in account the interestingness.

- $\quad \operatorname{probor}(\min (\mathbf{A}, \mathbf{B}), \mathbf{C})$ : takes the less value between rule quality and rule interpretability but may ignore any of the values, and also takes in account the interestingness.

- $\quad \operatorname{probor}(\max (\mathbf{A}, \mathbf{B}), \mathbf{C})$ : takes the highest value between rule quality and rule interpretability but will not ignore any of the values, and also takes in account the interestingness. When a rule has a good interestingness value, it's fitness value will arise.

The $\operatorname{probor}(\min (\mathbf{A}, \mathbf{B}), \mathbf{C})$ has the best effect on the fitness function, so this research adopted this function as a fitness function for the GA.

\section{EXPERIMENT AND RESULTS}

A genetic fuzzy rule-based system decreases the amount of knowledge and effort required from an expert in designing the inference system design. Evolutionary computation automates the design steps of the FRBS through rule learning. The GA here is the machine learning tool.

The fitness function, also known as the objective function, gives the measurement of the chromosome's ability to meet the desired criteria and is employed in global and local search methods.

Through computational experiments on the AFR (Air/Fuel Ratio) data sets, the new composition function is applied.

The data was collected from University Science Malaysia engines laboratory using a CB 125cc air cooled 4-stroke engine from Taiwan manufacturer 'Adly' equipped with a Euro-3 compliant Focus Applied Technologies gasoline EFI system and 3-way catalyst. The AFR data sets are a total of three data sets which are used for the research. This number includes engine operation of 15,182 samples selected from the chosen dataset's testing and training information for the purpose of achieving firm temporary states.

The scope of the data factors is as follows: MAP [0 - 1.05] bar, TPS [0\% - 194.49\%], RPM [0 - 8900] revolution per minute, MAT $\left[26.7 \mathrm{C}^{\circ}-34.4 \mathrm{C}^{\circ}\right.$, CLT $\left[29 \mathrm{C}^{\circ}-141 \mathrm{C}^{\circ}\right.$, and PW [0.6ms - 7.9ms]. Table 4 depicts he characteristics of the experimental data sets.

Table 4: Characteristics of the Experimental Data Sets

\begin{tabular}{ccc}
\hline Data Sets & Number of Examples & Number of Factors \\
\hline Data Set1 & 3500 & 6 \\
Data Set2 & 5776 & 6 \\
Data Set3 & 5906 & 6 \\
\hline
\end{tabular}


The GA takes its values of genes from the data sets directly. But while we are using fuzzy logic as a way of dealing with uncertainty of the sensors producing the data, the encoding of the individual of GA is applied using the membership function for each fuzzy set. As the inputs are represented by more than one fuzzy set, the FCM decides the number of fuzzy sets where each cluster stands for a fuzzy set that are overlapped by the fuzzy nature. So each input can be represented by $n$ of membership grades produced by applying the Gaussian membership function.

Figure 3, 4, 5 present examples of the fuzzy rules resulted from applying the fuzzy GA algorithm on the Data Set1, Data Set2, and Data Set 3 respectively with 3-objectives .

Rule1: If (in1 is in1cluster4) and (in2 is in2cluster11) and (in6 is in6cluster15) then (out1 is out1cluster10)

Rule2: If (in1 is in1cluster4) and (in2 is in2cluster9) and (in6 is in6cluster15) then (out1 is out1cluster12)

Rule3: If (in2 is in2cluster9) and (in4 is in4cluster6) and (in5 is in5cluster17) then (out1 is out1cluster12)

Rule4: If (in4 is in4cluster14) and (in5 is in5cluster13) then (out1 is out1cluster5)

Rule5: If (in1 is in1cluster17) and (in2 is in2cluster10) and (in3 is in3cluster16) then (out1 is out1cluster4)

Rule6: If (in1 is in1cluster2) and (in2 is in2cluster16) and (in4 is in4cluster14) and (in5 is in5cluster5) then (out1 is out1cluster4)

Rule7: If (in1 is in1cluster3) and (in2 is in2cluster4) and (in6 is in6cluster2) then (out1 is out1cluster13)

Rule8: If (in1 is in1cluster5) and (in3 is in3cluster15) and (in4 is in4cluster8) and (in5 is in5cluster8) then (out1 is out1cluster8)

Rule9: If (in2 is in2cluster5) and (in4 is in4cluster7) and (in6 is in6cluster5) then (out1 is out1cluster7)

Rule10: If (in1 is in1cluster17) and (in2 is in2cluster1) then (out1 is out1cluster7)

Rule11: If (in4 is in4cluster7) and (in5 is in5cluster5) and (in6 is in6cluster2) then (out1 is out1cluster16)

Rule12: If (in1 is in1cluster5) and (in2 is in2cluster12) and (in6 is in6cluster3) then (out1 is out1cluster11)

Rule13: If (in1 is in1cluster11) and (in3 is in3cluster3) and (in4 is in4cluster1) and (in5 is in5cluster7) and (in6 is in6cluster9) then (out1 is out1cluster2)

Rule14: If (in1 is in1cluster2) and (in5 is in5cluster13) and (in6 is in6cluster11) then (out1 is out1cluster9)

Rule15: If (in2 is in2cluster5) and (in4 is in4cluster1) and (in5 is in5cluster11) then (out1 is out1cluster7)

Rule16: If (in 2 is in2cluster17) and (in 4 is in 4cluster1) and (in 5 is in5cluster2) then (out1 is out1cluster14)

Rule17: If (in1 is in1cluster11) and (in2 is in2cluster17) and (in3 is in3cluster2) then (out1 is out1cluster10)

Rule18: If (in1 is in 1cluster15) and (in 2 is in2cluster14) and (in 3 is in 3 cluster1) then (out1 is out1cluster8)

Figure 3. Fuzzy Rules of Proposed Algorithm for Data Set1

Rule1: If (in1 is in1cluster9) and (in2 is in2cluster9) and (in3 is in3cluster3) then (out1 is out1cluster4)

Rule2: If (in2 is in2cluster9) and (in3 is in3cluster1) and (in6 is in6cluster3) then (out1 is out1cluster4)

Rule3: If (in3 is in 3cluster3) and (in 4 is in4cluster5) and (in5 is in5cluster7) then (out1 is out1cluster5)

Rule4: If (in1 is in1cluster2) and (in4 is in4cluster2) and (in5 is in5cluster9) then (out1 is out1cluster9)

Rule5: If (in1 is in1cluster8) and (in4 is in4cluster4) and (in5 is in5cluster2) and (in6 is in6cluster1) then (out1 is out1cluster6)

Rule6: If (in2 is in2cluster6) and (in3 is in3cluster6) and (in6 is in6cluster2) then (out1 is out1cluster3)

Rule7: If (in3 is in3cluster1) and (in4 is in4cluster8) and (in6 is in6cluster9) then (out1 is out1cluster8)

Rule8: If (in2 is in2cluster3) and (in3 is in3cluster3) and (in5 is in5cluster4) then (out1 is out1cluster2)

Rule9: If (in3 is in3cluster2) and (in4 is in4cluster6) then (out1 is out1cluster8) (1)

Rule10: If (in1 is in1cluster5) and (in2 is in2cluster5) and (in4 is in4cluster4) and (in5 is in5cluster2) then (out1 is out1cluster7)

Rule11: If (in1 is in1cluster9) and (in3 is in3cluster1) and (in4 is in4cluster2) and (in5 is in5cluster7) then (out1 is out1cluster6)

Rule12: If (in1 is in1cluster6) and (in2 is in2cluster6) then (out1 is out1cluster5)

Rule13: If (in3 is in3cluster9) and (in4 is in4cluster4) and (in5 is in5cluster5) then (out1 is out1cluster3)

Rule14: 14. If (in1 is in1cluster4) and (in 2 is in2cluster4) and (in 4 is in4cluster8) then (out1 is out1cluster1)

Figure 4. Fuzzy Rules of Proposed Algorithm for Data Set2

Rule1: If (input 1 is $\mathrm{mf} 1$ ) and (input 2 is $\mathrm{mf} 1$ ) then (output 1 is $\mathrm{mf} 4$ )

Rule2: If (input3 is $\mathrm{mf} 5$ ) and (input4 is $\mathrm{mf} 3$ ) and (input5 is $\mathrm{mf} 6$ ) then (output 1 is $\mathrm{mf} 3$ )

Rule3: If (input 3 is $\mathrm{mf} 2$ ) and (input4 is $\mathrm{mf} 4$ ) and (input5 is $\mathrm{mf} 5$ ) then (output1 is $\mathrm{mf} 6$ )

Rule4: If (input3 is $\mathrm{mf} 6$ ) and (input4 is $\mathrm{mf} 5$ ) and (input5 is $\mathrm{mf} 1$ ) then (output 1 is $\mathrm{mf} 1$ )

Rule5: If (input 1 is $\mathrm{mf} 4$ ) and (input 3 is $\mathrm{mf} 4$ ) and (input 4 is $\mathrm{mf} 7$ ) and (input5 is $\mathrm{mf} 6$ ) then (output 1 is $\mathrm{mf} 7$ )

Rule6: If (input2 is $\mathrm{mf} 4$ ) and (input 3 is $\mathrm{mf} 4$ ) and (input4 is $\mathrm{mf} 7$ ) and (input5 is $\mathrm{mf} 8$ ) then (output 1 is $\mathrm{mf} 7$ )

Rule7: If (input3 is $\mathrm{mf} 6$ ) and (input4 is $\mathrm{mf} 4$ ) and (input5 is $\mathrm{mf} 3$ ) then (output1 is $\mathrm{mf} 4$ )

Rule8: If (input1 is mf5) and (input2 is mf5) then (output1 is mf2)

Rule9: If (input1 is $\mathrm{mf} 3$ ) and (input 3 is $\mathrm{mf} 6$ ) and (input4 is $\mathrm{mf} 4$ ) and (input5 is mf6) then (output1 is mf1)

Rule10: If (input 3 is $\mathrm{mf} 1$ ) and (input4 is $\mathrm{mf} 1$ ) and (input5 is $\mathrm{mf} 5$ ) then (output 1 is $\mathrm{mf} 2$ )

Rule11: If (input2 is $\mathrm{mf} 4$ ) and (input 3 is $\mathrm{mf} 1$ ) and (input4 is $\mathrm{mf} 1$ ) then (output 1 is $\mathrm{mf} 7$ )

Rule12: If (input1 is $\mathrm{mf} 5$ ) and (input 4 is $\mathrm{mf} 7$ ) and (input5 is $\mathrm{mf}$ ) then (output1 is $\mathrm{mf} 6$ )

Rule13: If (input 3 is $\mathrm{mf} 1$ ) and (input 4 is $\mathrm{mf} 5$ ) and (input5 is mf7) then (output 1 is $\mathrm{mf} 5$ )

Figure 5. Fuzzy Rules of Proposed Algorithm for Data Set3 
The Average number of rules and terms for each of the Proposed algorithm is presented in table 5 and 6.

Table 5: Average number of rules of Original algorithm and Proposed Algorithm

Data Sets $\quad$ Proposed Algorithm

Data Set1

Data Set2

Data Set3

Table 6: Average number of Terms of Original algorithm and Proposed Algorithm

Data Sets

Proposed Algorithm

Data Set1

Data Set2

42

Data Set3

40

\section{CONCLUSION}

In this work, for a multi-objective fitness function, we used a composition function to aggregate the objectives without the need for weights. The objectives are conflicting in nature such as the accuracy and interpretability. Trying to avoid this problem, we took the inverse of the interpretability value in Equation 2. The function tries to make a trade-off between the objectives.

In this research, we noticed that the probor(min) is the best compared with others to apply the fitness function. We can notice that depending on the first part from of the function, the second part gives an importance to the interestingness which we require. The fitness of a rule with low interestingness, though it has high quality or interpretability, it will be weaken depending on the degree of interestingness of the rule. But if it has high interestingness, the fitness of the rule will be supported. Probor function is the most that supports this theory.

\section{REFERENCES}

- $\quad$ Dehuri, S., Patnaik, S., Ghosh, A., \& Mall, R. (2008). Application of elitist multi-objective genetic algorithm for classification rule generation. Applied soft computing, 8(1), 477-487.

- Fernández, A., López, V., del Jesus, M. J., \& Herrera, F. (2015). Revisiting Evolutionary Fuzzy Systems: Taxonomy, applications, new trends and challenges. Knowledge-Based Systems.

- Gacto, M. J., Alcalá, R., \& Herrera, F. (2010). Integration of an index to preserve the semantic interpretability in the multiobjective evolutionary rule selection and tuning of linguistic fuzzy systems. Fuzzy Systems, IEEE Transactions on, 18(3), 515-531.

- Gacto, M. J., Alcalá, R., \& Herrera, F. (2011). Interpretability of linguistic fuzzy rule-based systems: An overview of interpretability measures. Information Sciences, 181(20), 4340-4360.

- Huysmans, J., Baesens, B., \& Vanthienen, J. (2006). Using rule extraction to improve the comprehensibility of predictive models. Available at SSRN 961358.

- Huysmans, J., Dejaeger, K., Mues, C., Vanthienen, J., \& Baesens, B. (2011). An empirical evaluation of the comprehensibility of decision table, tree and rule based predictive models. Decision Support Systems, 51(1), $141-154$

- Ishibuchi, H., Nakashima, T., \& Murata, T. (2001). Three-objective genetics-based machine learning for linguistic rule extraction. Information Sciences, 136(1), 109-133. 
- Ishibuchi, H., \& Nojima, Y. (2007). Analysis of interpretability-accuracy tradeoff of fuzzy systems by multiobjective fuzzy genetics-based machine learning. International Journal of Approximate Reasoning, 44(1), 4-31.

- Ishibuchi, H., \& Yamamoto, T. (2004). Fuzzy rule selection by multi-objective genetic local search algorithms and rule evaluation measures in data mining. Fuzzy Sets and Systems, 141(1), 59-88.

- Mandal, S., \& Pal, M. K. K. S. K. (2011). Pattern Recognition and Machine Intelligence.

- Noda, E., Freitas, A., \& Lopes, H. S. (1999). Discovering interesting prediction rules with a genetic algorithm. Paper presented at the Evolutionary Computation, 1999. CEC 99. Proceedings of the 1999 Congress on.

- Noda, E., Freitas, A. A., \& Yamakami, A. (2003). A Distributed-Population GA for Discovering Interesting Prediction Rules Advances in Soft Computing (pp. 287-296): Springer.

- $\quad \mathrm{Xu}$, J., \& Zhou, X. (2011). Fuzzy-like multiple objective decision making (Vol. 263): Springer. 